\title{
1 What does first-line therapy mean for paediatric 2 multiple sclerosis in the current era?
}

3 Yael Hacohen, Brenda Banwell and Olga Ciccarelli

4

5 Yael Hacohen

6 Department of Neuroinflammation, Queen Square MS Centre, UCL Institute of Neurology, University

7 College London, London, UK/Department of Paediatric Neurology, Great Ormond Street Hospital for

8 Children, London, UK

9 Brenda Banwell

10 Division of Neurology, Department of Pediatrics, Children's Hospital of Philadelphia, Philadelphia,

11 PA, USA/Department of Neurology, Perelman School of Medicine, University of Pennsylvania,

12 Philadelphia, PA, USA

13 Olga Ciccarelli

14 Department of Neuroinflammation, Queen Square MS Centre, UCL Institute of Neurology, University

15 College London, London, UK/NIHR UCLH Biomedical Research Centre, London, UK 


\section{Abstract}

2 Paediatric multiple sclerosis (MS) is associated with higher relapse rate, rapid MRI lesion accrual early in the disease course and worse cognitive outcome and physical disability in the long term compared to adult-onset disease. Current treatment strategies are largely centre-specific and reliant on adult protocols. The aim of this review is to examine which treatment options should be considered first line for paediatric MS and we attempt to answer the question if injectable first-line DMTs are still an optimal option. To answer this question, we review the effects of early onset disease on clinical course and outcomes, with specific considerations on risks and benefits of treatments for paediatric MS. Considering the impact of disease activity on brain atrophy, cognitive impairment and development of secondary progressive MS at a younger age we would recommend treating paediatric MS as a highly-active disease, favouring the early use of highly effective DMTs rather than injectable DMTs.

\section{Introduction}

The approach to treatment of relapsing remitting multiple sclerosis (RRMS) in children is rapidly evolving as clinicians consider paediatric indications for the now increasing number of diseasemodifying therapies (DMTs) currently licensed for adults ${ }^{1}$.

In this review, we weigh the now sizeable body of data that supporting that POMS is associated with a high degree of clinical and MRI inflammatory disease activity and with early loss of brain tissue against the modest efficacy of injectable conventionally-first-line disease modifying therapies (DMTs). We discuss the risks of MS disease-damage, with special consideration of such damage in the maturing brain and compare this to therapeutic risks in (i) lower potency but systemically safe therapies such as interferons or glatiramer acetate (GA) which are likely to incompletely mitigate MS disease activity and (ii) higher potency therapies which are superior in mitigating MS disease activity but have higher known and conceptual risks compared to lower potency DMTs.

With the paucity of clinical trial evidence for all but a few of the current MS therapies, treatment selection for paediatric MS patients rests on provider- and centre-specific practice and extrapolation from adult MS therapeutic protocols for specific medications. Clinical trials in the paediatric population, as well as surveillance programs to monitor for long-term impact of therapies on somatic growth, fertility and secondary autoimmune or oncologic complications are essential in order to inform practice. Achieving these imperatives, however, is challenged by the rarity of paediatric-onset MS (POMS), and the even smaller number of paediatric MS patients exposed to any one specific therapy. Long-term monitoring requires collaboration between paediatric and adult MS 
1 providers in order to chart the clinical course of such patients over a sufficient window of

2 observation. As is true for adult MS, risks and toxicities relate not only to an individual therapy, but

3 also to the cumulative array of therapeutic exposures, and potential additive risks related to chronic

4 treatment. Critical to meaningfully appreciating the benefit of early MS care will be to develop

5 patient-centred outcomes that are sensitive to early physical impairments and that novel measures

are developed to assess cognitive, social and mental health outcomes.

7 MRI has proven invaluable in the evaluation of treatment efficacy in adults with MS, with

8 suppression of new or newly enlarging T2 and new gadolinium enhancing lesions being the primary

9 metrics indicative of inflammatory disease control. In paediatric MS, there exists the additional

10 considerations relating to the negative impact of MS in the maturing CNS.

11 Fundamental to the diagnosis of MS in children, as in adults, is to exclude other diagnoses. Exclusion

12 of antibody-mediated disease (e.g., myelin oligodendrocyte glycoprotein (MOG) and acquaporin-4

13 (AQP4) antibodies) ${ }^{2}$ and consideration of monogenetic disorders with relapsing remitting clinical

14 course, such as primary central nervous system-hemophagocytic lymphohistiocytosis,

15 leukodystrophies and mitochondrial disease, must be carefully considered for all patients being

16 diagnosed with MS.

\section{Clinical and radiological features and outcomes of paediatric-onset MS}

18 Paediatric MS has an incidence ranging between 0.13 and 0.66 per 100,000 children per year ${ }^{3}$. The median age at presentation is 13.7 years ${ }^{4}$ and although isolated reports of MS presenting in young prepubertal children, the majority of paediatric patients are teenagers. POMS is associated with a high relapse rate ${ }^{5}$, rapid MRI lesion accrual early in the disease course ${ }^{6}$ risk for adverse cognitive outcomes $^{7}$ and potentially with the risk for physical disability in the long term ${ }^{8}$.

Despite a highly active disease, particularly in the initial years, patients with POMS demonstrate a slower rate of accrual of disability compared to adult-onset patients. In a comparative study between 710 patients with adult onset MS and 83 with POMS, the Expanded Disability Status Scale (EDSS) evaluated at last clinical examination (mean 5 years) was lower in the paediatric onset group, despite a longer disease duration ${ }^{9}$. In a study of 59 paediatric MS patients followed for a median of 5.9 years, $90 \%$ continued to have a normal neurological examination ${ }^{10}$. In a German study of 88 children with MS, the median EDSS scores were less than 1 at 2 years, 1.2 at 10 years and 2.5 at 15 years $^{11}$. A seminal paper which compared 394 patients with paediatric onset MS to 1775 patients with adult onset MS demonstrated that it took approximately 10 years longer for the paediatric-onset patients to reach irreversible disability and SPMS; however, they reached these landmarks at a biological age approximately 10 years younger than their counterparts with adults- 
1 onset disease ${ }^{8}$. In this cohort, the median time to conversion to secondary progressive disease was 28.1 years.

Data from population-based longitudinal cohort study from Swedish MS Registry evaluating cognitive outcome of 5704 patients with MS (300 of whom had paediatric-onset disease) ${ }^{12}$ revealed that paediatric-onset patients had greater deficits in information processing, measured by the Symbol Digit Modalities Test (SDMT) than their counterparts with adult-onset disease, independent of age or disease duration.

Brain volume is reduced in paediatric-onset MS when compared to healthy controls, and this reduction in volume is evident at clinical presentation ${ }^{13-15}$. Brain atrophy accelerates over the first 2 years and is linked to disease activity ${ }^{14}$. Interestingly, in the early stages of the disease, despite the development of brain atrophy, neither physical nor cognitive deficits are typically present ${ }^{13}$. As will be discussed below, rate of brain volume loss can be partially mitigated by effective suppression of relapsing disease ${ }^{16}$.

\section{Disease modifying treatments (DMTs)}

Prior to 2018, when fingolimod was the first drug to be approved by the Food and Drug Administration (FDA) and the European Medicines Agency (EMA), paediatric neurologists had used DMTs off label to treat children with MS. In 2012, the IPMSSG guidelines recommended early initiation of DMTs with either an interferon $\beta$ or glatiramer acetate as first-line therapy ${ }^{17}$. The majority of data comes from open-label observational or retrospective studies, ${ }^{18}$ which suggest a reduced annual relapse rate compared to pre-treatment ${ }^{19,20}$ ranging from a pre-treatment annualised relapse rate of 1.9-3.2 to 0.04-0.9 after treatment initiation ${ }^{21}$.

Timing of treatment is also key; a study from the Danish Multiple Sclerosis Registry compared POMS patients who were commenced on DMTs (76\% of patients treated with interferon $\beta$ ) within 2 years of onset $(N=140)$ or later $(N=151)$ and demonstrated that starting on a DMT later had a 2.52-fold increased risk of reaching sustained EDSS 4 compared to starting within 2 years of onset $(H R=2.52$, 95\% confidence interval $(\mathrm{Cl})=1.01-6.34)$. For every year increment from onset to start of first DMT, the risk of reaching sustained EDSS 4 increased by $17 \%(\mathrm{HR}=1.17,95 \% \mathrm{Cl}=1.05-1.30)^{22}$.

A high rate of treatment failure with injectable treatments has been reported in paediatrics, and this ranged from $25 \%$ to $64 \%$ across studies ${ }^{21}$. In a multicentre, retrospective, longitudinal, open-label study of 258 patients with paediatric-onset $\mathrm{MS}^{23}$ with a mean follow-up of 3.9 years, $44 \%$ of patients experienced refractory disease (defined as the presence of clinical activity and/or MRI activity) on their first DMT. The majority were switched to another injectable with a different mechanism of action (ie: interferon to GA, or vice versa), and $20 \%$ moved on to second-line therapies. In a more 
1 recent study of 97 paediatric MS patients with a longer follow-up duration of $12.5 \pm 3.3$ years ${ }^{19}, 82$

2 (84.5\%) changed therapies. Compared to pre-treatment phase, the annualised relapse rate was

3 significantly reduced during the first treatment (from $3.2 \pm 2.6$ to $0.7 \pm 1.5, p<0.001$ ), and it

4 remained low during the whole follow-up $(0.3 \pm 0.2, p<0.001)$. In an observational multicentre study

5 from the US Network of Paediatric MS Centres of 618 patients receiving DMTs ${ }^{24}, 483(78 \%)$ received

6 injectable treatments first line; of these 147 (30.4\%) switched to therapies. The DMTs most

7 frequently used in this cohort were dimethyl fumarate $(n=102)$ followed by natalizumab $(n=101)$,

8 rituximab $(n=57)$ and fingolimod $(n=37)$. At a mean follow-up of $3.5( \pm 3.1)$ years, DMTs had a similar

9 short-term safety, tolerability, and side effect profiles as in adults. In a more recent study from the

10 US Network of Paediatric MS Centres of 741 patients (under the age of 18years), 197 where

11 commenced on newer therapies (fingolimod, dimethyl fumarate, teriflunomide, natalizumab,

12 rituximab, ocrelizumab) and 544 on injectable DMTs (interferon- , glatiramer acetate) ${ }^{25}$. Following

13 propensity scores-quintile adjusted analysis, those on newer DMTs had lower relapse rate than

14 those on injectables (rate ratio $0.45,95 \% \mathrm{Cl} 0.29-0.70, p<0.001$; rate difference $0.27,0.14-0.40$,

$15 \mathrm{p}=0.004)^{25}$ suggesting a favourable treatment effect of newer therapies.

16 Fundamental to efficacy of any therapy is adherence. In a study of paediatric MS patients, self-

17 reported non-adherence (not taking the medication for $>20 \%$ of doses in the past month) was as

18 high as $37 \%{ }^{26}-41 \%{ }^{27}$. Importantly, lack of adherence was similar between oral and injectable

19 therapies; infusion-based treatments were not evaluated as such treatments were less commonly

20 used at the time of these manuscripts. An important consideration in regards to adherence, relates

21 to patient autonomy. Oral DMTs were associated with lower levels of parental involvement in DMT

22 administration, which is an important factor to consider in adolescents who place high value on their

23 personal perception of self-control. ${ }^{25}$ It will be important to evaluate adherence in patients treated

24 with twice annual infusion protocols, such as prescribed for anti-CD20 therapies, or even monthly

25 infusions, as required for natalizumab, where adherence is facilitated by reduced frequency of

26 treatment and by the highly visible nature of pre-booked appointments.

27 A key consideration in regards to adherence is the risk of rebound of disease activity (clinical and

28 radiographic), including a risk of even more severe disease activity than prior to commencement of

29 therapy. These risks are particularly noted in patients who stop treatment with fingolimod ${ }^{28}$ or

30 natalizumab 29.

31 The results of the first randomised controlled trial of fingolimod versus interferon $\beta$ 1a in paediatric

32 MS were recently published ${ }^{30}$. Of a total of 215 patients, 107 were assigned to fingolimod and 108 to

33 interferon beta-1a. The mean age of the patients was 15.3 years with a mean of 2.4 relapses during

34 the preceding 2 years. The study clearly demonstrated the superiority of fingolimod with a lower 
1 rate of relapse ( 0.12 vs $0.67, p<0.001)$, less accumulation of lesions on MRI (4.39 vs $9.27, P<0.001)$

2 and a lower annualized rate of brain atrophy ${ }^{16}$ over a 2-year period than interferon ${ }^{30}$.

3 In adult relapsing remitting MS studies, highly potent agents such as alemtuzumab, cladribine and

4 autologous haematopoietic stem cell transplantation have demonstrated complete suppression of clinical and MRI new disease. While highly effective, the risks of profound immunosuppression limit wide-spread application of these therapies, and these treatments have not been studied in children.

7 The highly inflammatory nature of POMS renders these young patients as potentially excellent candidates for these potent therapies, but the risk profile remains a serious barrier. Higher risk therapies are the mainstay of oncology therapy in paediatrics; however, the disease risk profile is clearly much different than that of MS in terms of mortality. Thus, while a great deal can be learned about risks of secondary infections and malignancies in paediatric populations exposed to profound immunosuppression, the risk to benefit ratio will remain a major challenge to the justification of such therapies in the context of paediatric MS.

\section{Safety: Infections, toxicity, risk of malignancies and vaccinations}

Data regarding the long-term impact of paediatric MS treatment on key outcomes such as risks for systemic illness, oncological diagnoses, fertility and on MS-specific outcomes such as age- and year post diagnosis at onset of secondary progressive MS and disability is currently unknown. Obtaining such data is inherently limited by the rarity of paediatric MS, by the need to international collaborative cohort data collection, and by the paucity of clinical trials with phase 4 extension studies. Table 1 provide a summary of key consideration in paediatrics.

First line injectable DMTs, interferon $\beta$ and glatiramer acetate (GA) are not associated with increased risks of infections or malignancies. To date, no life-threatening events or mortalities have been reported in children or adolescents exposed to interferon or GA. Infection risk is a greater consideration with new therapies. Among specific infections, Varicellazoster virus (VZV) has been specifically associated with fingolimod, but overall, in clinical trials, VZV rates of infection were low but higher with fingolimod compared with placebo (11 vs 6 per 1000

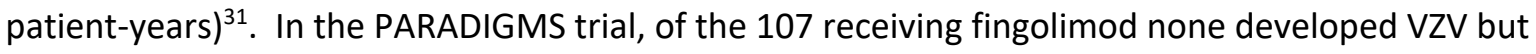
other infections (appendicitis, cellulitis, gastrointestinal infection, oral abscess, viral infection, and viral pharyngitis were reported in four patients $(3.7 \%)$ compared to $2 / 108$ (1.9\%, paronychia and viral gastritis)) reported infections in the interferon beta-1a group. The fingolimod dose for children under $40 \mathrm{~kg}$ was $0.25 \mathrm{mg}$ (vs $0.5 \mathrm{mg}$ for over $40 \mathrm{~kg}$ ). As only 10/215 children were less than $40 \mathrm{~kg}$ in this cohort (9 patients in the fingolimod group), it was not possible to evaluate the effect of dose and age on the risk of infections. Natalizumab increases the risk for life-threatening progressive 
1 multifocal leukoencephalopathy (PML) in MS patients who have JC virus. The age at which primary

2 JC virus infection occurs is variable, with approximately $60 \%$ of persons testing positive by age 30

3 years. As such, only $10 \%$ of 10 years-old children and $50 \%$ of adolescents are seropositive ${ }^{32}$. Use of

4 natalizumab in JC virus-negative patients clearly does not carry the risk of PML, provided that careful monitoring for seroconversion occurs while on therapy. Treatment with anti-CD 20 molecules, such as ocrelizumab, is associated with a higher rate of respiratory tract infections when compared with interferon $\beta$ and placebo in adult patients ${ }^{33}$, but no paediatric data are available. Experience from rituximab (also anti-CD20) suggests a possible increased frequency of hypogammaglobulinemia in children compare to adults ${ }^{34}$.

The emergence of novel coronavirus 2019 (COVID-19) pandemic presented a new challenge to neurologists managing children with MS. Not only has the pandemic limited in person clinical care visits, imaging and access to laboratory monitoring, it has prompted concern about whether COVID19 will results in increased morbidity or mortality in the MS population. Expert opinion posits that interferons and GA, which are not associated with higher infection rates in general, should be continued at normal dosing. Therapies that reduce lymphocyte number, lymphocyte trafficking, and function could theoretically predispose to a greater risk for symptomatic and a potentially more severe COVID-19 infection ${ }^{35}$. It is also conceivable that immunosuppression may confer a reduced risk for the inflammatory response to SARS-CoV2. While ongoing surveillance and global reporting of the impact of COVID-19 in person with MS is ongoing across the world, general guidance for paediatric MS advises that the benefits of continuing MS treatment outweigh the risks of stopping therapy and potentially experiencing new relapses. This advice is based on the following: (i) children with MS have a high relapse rate, and sudden cessation of MS therapies is highly likely to result in a clinical attack; (ii) paediatric MS patients rarely have comorbid pulmonary disease, hypertension, or diabetes, risk factors associated with higher morbidity in COVID-19 disease ${ }^{36}$; and (iii) paediatric MS patients are rarely disabled, rarely have entered secondary progressive disease, and none have primary progressive MS- risk factors identified for higher morbidity in the adult MS population. In alignment with international guidelines, initiation of MS therapy during the pandemic might reasonably avoid therapies known to result in profound immunosuppression, such as alemtuzimab, cladribine or haemopoietic stem cell transplantation- therapies that are currently rarely, used in children. Worrisome is the emergence of Multisystem Inflammatory Disease in Children (MIS-C) ${ }^{37}$, which has not yet been reported in paediatric MS patients infected with SARS-CoV2. teenagers will require treatment for decades. Long-term risks will be influenced by immunological mechanism of action of therapies provided, the total number of therapies used, and sequence of 
treatment, and by the development of co-morbidities (smoking, obesity, hypertension, diabetes, for example) that occur during the lifetime. A critical facet of care for all persons with MS is to promote wellness strategies, such as avoidance of smoking, vaping or inhalants, healthy weight, exercise, vitamin D supplementation, and mental health supports.

Vaccinations are an important facet of paediatric health care, and childhood is a period where the frequency of required vaccines is higher than in adulthood. The potential for a SARS-CoV2 vaccine is also a key hope for the coming years. While comprehensive analysis of the safety and immunogenicity of specific vaccinations in paediatric MS is not available, several key guiding principles apply: (i) prior to commencing MS therapy, all required primary vaccines should be administered; (ii) vaccination against varicella or proof of immunity should be ensured; (iii) pneumococcal vaccination is recommended in most countries prior to initiation with anti-CD20 therapy; and (iv) the vaccine for human papilloma virus should be discussed with both female and male patients. Live and live-attenuated vaccines should not be given to patients who are actively treated. Administration of inactivated vaccines can occur during therapy, including anti-CD20 treatments, although the immunogenicity is likely reduced.

\section{Conclusions and future steps}

17 The onset of MS during childhood or adolescence is associated with frequent clinical relapses, with 18 the accrual of a high volume of T2 lesions, with progressive loss of brain tissue, and with a risk over the subsequent decades for progressive cognitive and physical disability. Interferons and GA offer modest therapeutic efficacy in adult clinical trials, and have been shown to have a favourable safety profile in paediatric and adult MS. However, the extent to which these therapies can effectively suppress MS disease activity, and the lack of adherence relating to injection fatigue seriously limit advocacy for these therapies in this new era of more effective treatments that are given by infusion or orally.

The current array of MS therapies offers multiple high potency options that very clearly reduce inflammatory disease activity and show promise for limiting brain atrophy- both in adults, and more recently demonstrated in the first Phase 3 therapeutic trial of fingolimod in children ${ }^{16}$. Long-term safety data is pivotal, and collaborative data collection would advance understanding of the use of MS therapies in the real world.

When considering all of these factors, one pivotal point is the importance of early effective therapy. Relapses are highest and brain tissue loss is rapid in the first years post-onset, and as such, we propose that the absolute critical window for the use of highly effective therapies is during this time period. We therefore posit that "first line" treatment for paediatric MS should be adjudicated by its 
ability to markedly suppress relapses and MRI new lesions and to preserve age-expected brain

growth and volume

\section{Financial Disclosure}

5 Yael Hacohen is funded by the UK MS society. She has no financial disclosures

Brenda Banwell serves as a consultant for Novartis, UCB Pharmaceuticals, and Roche. Dr. Banwell

provides non-remunerated consultative support to Teva Neuroscience, Novartis, Biogen, and

8 Genentech. Dr. Banwell is funded by the National MS Society, NIH, and Multiple Sclerosis Society of

9 Canada

10 Olga Ciccarelli receives research funding from UK MS Society, Rosetrees trust and NIHR UCLH BRC

11 and serves as a consultant for Biogen, Novartis, Roche, Genzyme, Teva and GE healthcare. She is on

12 the editorial board of neurology.

\section{References}

1. Thompson AJ, Baranzini SE, Geurts J, Hemmer B, Ciccarelli O. Multiple sclerosis. Lancet 2018;391:1622-1636.

18 2. Hacohen Y, Mankad K, Chong WK, et al. Diagnostic algorithm for relapsing acquired 19 demyelinating syndromes in children. Neurology 2017;89:269-278. 3. Waldman A, Ghezzi A, Bar-Or A, Mikaeloff Y, Tardieu M, Banwell B. Multiple sclerosis in children: an update on clinical diagnosis, therapeutic strategies, and research. Lancet Neurol2014: 936-948.

4. Hacohen Y, Brownlee W, Mankad K, et al. Improved performance of the 2017 McDonald criteria for diagnosis of multiple sclerosis in children in a real-life cohort. Mult Scler 2019:1352458519863781.

5. Gorman MP, Healy BC, Polgar-Turcsanyi M, Chitnis T. Increased relapse rate in pediatric-onset compared with adult-onset multiple sclerosis. Arch Neurol 2009;66:54-59. 6. Waubant $E$, Chabas D, Okuda DT, et al. Difference in disease burden and activity in pediatric patients on brain magnetic resonance imaging at time of multiple sclerosis onset vs adults. Arch Neurol 2009;66:967-971.

7. Baruch NF, O'Donnell EH, Glanz BI, et al. Cognitive and patient-reported outcomes in adults with pediatric-onset multiple sclerosis. Mult Scler 2016;22:354-361.

8. Renoux C, Vukusic S, Mikaeloff Y, et al. Natural history of multiple sclerosis with childhood onset. N Engl J Med 2007;356:2603-2613.

9. Simone IL, Carrara D, Tortorella C, et al. Course and prognosis in early-onset MS: comparison with adult-onset forms. Neurology 2002;59:1922-1928.

10. O'Mahony J, Marrie RA, Laporte A, et al. Recovery From Central Nervous System Acute Demyelination in Children. Pediatrics 2015;136:e115-123.

39 11. Huppke B, Ellenberger D, Rosewich H, Friede T, Gartner J, Huppke P. Clinical

40 presentation of pediatric multiple sclerosis before puberty. Eur J Neurol 2014;21:441-446. 

Cognitive Outcomes in Patients With Pediatric-Onset vs Adult-Onset Multiple Sclerosis. JAMA Neurol 2019.

4 13. De Meo E, Meani A, Moiola L, et al. Dynamic gray matter volume changes in 5 pediatric multiple sclerosis: A 3.5 year MRI study. Neurology 2019;92:e1709-e1723.

6 14. Aubert-Broche B, Fonov V, Narayanan S, et al. Onset of multiple sclerosis before 7 adulthood leads to failure of age-expected brain growth. Neurology 2014;83:2140-2146.

8 15. Bartels F, Nobis K, Cooper G, et al. Childhood multiple sclerosis is associated with 9 reduced brain volumes at first clinical presentation and brain growth failure. Mult Scler $10 \quad$ 2019;25:927-936.

11 16. Arnold DL, Banwell B, Bar-Or A, et al. Effect of fingolimod on MRI outcomes in patients with paediatric-onset multiple sclerosis: results from the phase 3 PARADIGMS study. J Neurol Neurosurg Psychiatry 2020.

17. Chitnis T, Tenembaum S, Banwell B, et al. Consensus statement: evaluation of new and existing therapeutics for pediatric multiple sclerosis. Mult Scler 2012;18:116-127. 18. Krupp LB, Tardieu M, Amato MP, et al. International Pediatric Multiple Sclerosis Study Group criteria for pediatric multiple sclerosis and immune-mediated central nervous system demyelinating disorders: revisions to the 2007 definitions. Mult Scler 2013;19:12611267.

19. Baroncini $D$, Zaffaroni M, Moiola L, et al. Long-term follow-up of pediatric MS patients starting treatment with injectable first-line agents: A multicentre, Italian, retrospective, observational study. Mult Scler 2018:1352458518754364.

20. Harding KE, Liang $\mathrm{K}$, Cossburn MD, et al. Long-term outcome of paediatric-onset multiple sclerosis: a population-based study. J Neurol Neurosurg Psychiatry 2013;84:141147.

21. Ghezzi A, Amato MP, Makhani N, Shreiner T, Gartner J, Tenembaum S. Pediatric multiple sclerosis: Conventional first-line treatment and general management. Neurology 2016;87:S97-S102.

22. Kopp TI, Blinkenberg M, Petersen T, Sorensen PS, Magyari M. Long term effect of delayed treatment on disability in patients with paediatric onset multiple sclerosis: A prospective Danish cohort study. Mult Scler Relat Disord 2020;40:101956.

23. Yeh EA, Waubant E, Krupp LB, et al. Multiple sclerosis therapies in pediatric patients with refractory multiple sclerosis. Arch Neurol 2011;68:437-444.

24. Krysko KM, Graves J, Rensel M, et al. Use of newer disease-modifying therapies in pediatric multiple sclerosis in the US. Neurology 2018;91:E1778-E1787.

25. Krysko KM, Graves JS, Rensel M, et al. Real-world effectiveness of initial diseasemodifying therapies in pediatric MS. Ann Neurol 2020.

26. Lulu S, Julian L, Shapiro E, Hudson K, Waubant E. Treatment adherence and transitioning youth in pediatric multiple sclerosis. Mult Scler Relat Disord 2014;3:689-695.

27. Schwartz CE, Grover SA, Powell VE, et al. Risk factors for non-adherence to diseasemodifying therapy in pediatric multiple sclerosis. Mult Scler 2018;24:175-185.

28. Hatcher SE, Waubant E, Nourbakhsh B, Crabtree-Hartman E, Graves JS. Rebound Syndrome in Patients With Multiple Sclerosis After Cessation of Fingolimod Treatment. JAMA Neurol 2016;73:790-794. 29. Vellinga MM, Castelijns JA, Barkhof F, Uitdehaag BM, Polman $\mathrm{CH}$. Postwithdrawal rebound increase in $\mathrm{T} 2$ lesional activity in natalizumab-treated MS patients. Neurology 2008;70:1150-1151. 
1 30. Chitnis T, Arnold DL, Banwell B, et al. Trial of Fingolimod versus Interferon Beta-1a in 2 Pediatric Multiple Sclerosis. N Engl J Med 2018;379:1017-1027.

3 31. Arvin AM, Wolinsky JS, Kappos L, et al. Varicella-zoster virus infections in patients

4 treated with fingolimod: risk assessment and consensus recommendations for

5 management. JAMA Neurol 2015;72:31-39.

6 32. Viscidi RP, Rollison DE, Sondak VK, et al. Age-specific seroprevalence of Merkel cell

7 polyomavirus, BK virus, and JC virus. Clin Vaccine Immunol 2011;18:1737-1743.

8 33. Hauser SL, Bar-Or A, Comi G, et al. Ocrelizumab versus Interferon Beta-1a in

9 Relapsing Multiple Sclerosis. N Engl J Med 2017;376:221-234.

10 34. Khojah AM, Miller ML, Klein-Gitelman MS, et al. Rituximab-associated

11 Hypogammaglobulinemia in pediatric patients with autoimmune diseases. Pediatr

12 Rheumatol Online J 2019;17:61.

13 35. Brownlee W, Bourdette D, Broadley S, Killestein J, Ciccarelli O. Treating multiple

14 sclerosis and neuromyelitis optica spectrum disorder during the COVID-19 pandemic.

15 Neurology 2020.

16 36. Richardson S, Hirsch JS, Narasimhan M, et al. Presenting Characteristics,

17 Comorbidities, and Outcomes Among 5700 Patients Hospitalized With COVID-19 in the New

18 York City Area. JAMA 2020.

19 37. Riphagen S, Gomez X, Gonzalez-Martinez C, Wilkinson N, Theocharis P.

20 Hyperinflammatory shock in children during COVID-19 pandemic. The Lancet. 


\begin{tabular}{|c|c|}
\hline & Paediatric consideration \\
\hline Interferon $\beta 1 a$ & \multirow[b]{2}{*}{$\begin{array}{l}\text { - Younger children AST/ALT elevation more prominent. Titrate more } \\
\text { slowly } \\
\text { - Risk of depression, which may be MS disease or therapy related and } \\
\text { may be more common in adolescent females } \\
\text { - Injection site reactions } \\
\text { - Body image concerns secondary to injection-related bruising, redness } \\
\text { - } \quad \text { Mor lipoatrophy } \\
\text { - }\end{array}$} \\
\hline Interferon $\beta 1 b$ & \\
\hline Glatiramer acetate & $\begin{array}{l}\text { - Body image concerns secondary to injection-related bruising, redness } \\
\text { or lipoatrophy } \\
\text { - More frequent parental involvement in injections }\end{array}$ \\
\hline Dimethyl Fumarate & $\begin{array}{l}\text { - } \quad \text { Compliance with twice daily dosing } \\
\text { - Facial flushing with morning dose (when going to school) } \\
\text { - Less need for parental involvement with oral medications }\end{array}$ \\
\hline Terifunomide & $\begin{array}{ll}\text { - } & \text { Teratogenicity } \\
\text { - } & \text { Hair thinning or loss } \\
\text { - } & \text { Less need for parental involvement with oral medications }\end{array}$ \\
\hline Fingolimod & $\begin{array}{l}\text { - Thymic maturation is largely completed in early childhood, but is still } \\
\text { ongoing through adolescence } \\
\text { - Concerns about long term malignancies } \\
\text { - No live vaccine while on treatment and reduced attenuated vaccine } \\
\text { efficacy } \\
\text { - Adherence and need for cardiac monitoring after missed doses (after } \\
\text { - } \text { missed dosing) }\end{array}$ \\
\hline Cladribine & $\begin{array}{l}\text { - More profound immunosuppression } \\
\text { - Weight based dosing with no data under } 40 \mathrm{~kg} \\
\text { - Live and live-attenuated vaccines are generally not recommended }\end{array}$ \\
\hline Ocrelizumab & $\begin{array}{l}\text { - Data from Rituximab may suggest increase risk of } \\
\text { hypogammaglobulineamia in children }\end{array}$ \\
\hline Natalizumab & $\begin{array}{l}\text { - Monthly infusions may impact school attendance } \\
\text { - Children more likely to be JC negative, rendering risk of PML extremely } \\
\text { low } \\
\text { - Adolescents may acquire primary JC infection highlighting importance } \\
\text { of surveillance }\end{array}$ \\
\hline Alemtuzumab & $\begin{array}{l}\text { - More profound immunosuppression } \\
\text { - Need for regular monthly white blood cell count, thyroid function, and } \\
\text { other laboratory tests; monitoring for at least } 5 \text { years } \\
\text { - Risk for autoimmune diseases } \\
\text { - Live and live-attenuated vaccines are generally not recommended }\end{array}$ \\
\hline
\end{tabular}


